\title{
Hepatitis A and E Virus Outbreaks in the Mediterranean Region 2006-2017: A Systematic Review
}

\section{Elena Charalambous*, Ioanna P Chatziprodromidou and Apostolos Vantarakis}

Department of Public Health, School of Medicine, University of Patras, Greece

*Corresponding Author: Elena Charalambous, Department of Public Health, School of Medicine, University of Patras, Greece.

Received: August 21, 2019; Published: October 11, 2019

DOI: $10.31080 /$ ASMI.2019.02.0392

\begin{abstract}
Objectives: A systematic review was conducted to improve understanding of the epidemiology of hepatitis A and E viruses (HAV,HEV), to investigate HAV and HEV viral outbreaks and to describe the distribution and magnitude of all cases in the Mediterranean region countries during the period 2006-2017.

Study Design: Not all study design types were included. Single case reports were not included unless the case was reported as the primary infection of the outbreak. This systematic review combined both experimental and non-experimental studies from 2006 onwards. Experts' opinions and qualitative studies were excluded.

Methods: Four databases (Scopus, PubMed, ISI Web of Science Core Correlation and SpringerLink electronic journal) were searched from 2006 to July 2017. Totally, 31 hepatitis A and E viral outbreaks in the Mediterranean region were identified. Most of the reported outbreaks were in European region, followed by South Mediterranean countries.

Results: Our research identified that the greatest number of studies (5.2\%) occurred in years 2013, 2008 and 2006 while $4.2 \%$ occurred in 2016 and 2010, 3.1\% in 2007 and 1.0\% in 2017, 2014, 2012, 2011 and 2009, respectively. The largest number of outbreaks (9.4\%) occurred in France, while 5.2\%, occurred in Spain, 4.2\% occurred in Egypt and Italy, 3.1\% occurred in Israel, 2.1\% occurred in Greece and 1.0\% occurred in Turkey, Algeria, Morocco and Tunisia respectively.

Conclusions: Hepatitis A and E are endemic in the Mediterranean region despite the subclinical features of the infections among residents; HAV and HEV conceal a danger to tourists and expatriates visiting the Mediterranean countries. The outbreaks, the frequency and the patterns of hepatitis viruses depict variations across the Mediterranean region depending on several demographic and socioeconomic factors. The prevalence of viral hepatitis infection is still high and consists a serious public health challenge.

Keywords: Hepatitis Virus; Hepatitis A; Hepatitis E; Outbreak; Mediterranean
\end{abstract}

\section{Abbreviations}

HAV: Hepatitis A Virus; HEV: Hepatitis E Virus.

\section{Introduction}

The prevalence of viral hepatitis is high and remains a serious public health challenge throughout the world [1,2]. An outbreak is commonly defined as the occurrence of cases of specific disease in a community with a frequency clearly in excess to normal expectancy [3].The number of cases indicating outbreak occurrence differs according to the infectious agent, the composition of the population exposed, previous experience or lack of exposure to the disease, as well as time and geographical coordinates of occurrence [4]. Therefore, the status of an outbreak is relative to the usual frequency of the disease in the same area, among the same population, at the same season of the year [5]. Certain food, such as ready to eat food (e.g., berries and shellfish) can themselves be a source of enteric viruses. In addition, outbreaks can often be foodborne, because of infection (including asymptomatic carriage) of a food handler. The food, which is affected by viruses, can be clas- sified into two distinct groups, based on the route of contamination. The first group includes vegetables, such as peppers, which can be contaminated with enteric viruses in their growing soil life [6-10] and the second group includes various kinds of food, other than vegetables, which are secondarily contaminated with enteric viruses, mainly by infected food handlers, during food processing and/or food serving [11]. Overall, food may be contaminated by a variety of ways, such as by faecal contaminated waters used to wash fruit and vegetables after harvest or used to irrigate the plants, the use of night soil to fertilise crops, or even by poor hand hygiene by an infected food handler $[12,13]$. Hepatitis A (HAV) is the cause of one of the most common forms of acute viral hepatitis in most parts of the world [14]. It still represents a public health problem in the Mediterranean region which is considered an area with high HAV prevalence [15]. HAV is a frequent travel acquired infection that has caused several outbreaks among European tourists $[16,17]$. HAV infection is largely acquired through fecal-oral transmission [18]. Since the major method of transmission is by person-to-person contact via the faecal-oral route, the sanitation 
and the quality of water supply are highly related with the prevalence of infection [19]. Nearly a third of patients acquire the infection from household or sexual contact with an infected person. Large families, poor education, inappropriate human-waste disposal system, crowded day-care centres and international travelling have been linked to outbreaks and endemicity of HAV infection [20]. However, approximately, $50 \%$ of the patients with hepatitis A do not have a recognized source of infection [21,22]. High-risk groups involve cleaning personnel in hospitals, staff in day-care centres, pediatric nurses, drug users, homosexuals, patients with chronic liver disease, international travellers and consumers of high-risk food (bivalve shellfish from stagnant water) [22]. Hepatitis E virus (HEV) consists of two species so far: (i) mammalian HEV, which causes human disease and infects several other mammalian species, in particular pigs; and (ii) avian HEV, which is known to infect birds such as turkeys [23]. Under genomic sequence analysis, human and animal HEV isolates are grouped into four genotypes, namely enotypes 1, 2, 3 and 4. A specific geographical distribution appears in each HEV genotype [24]. HEV is excreted in faeces of humans infected with this virus and is transmitted mainly through the faecal-oral route [25]. Transmission through contaminated water is the most common, while also zoonotic transmission of HEV appears to be common in hyperendemic areas [25]. The HEV genotypes prevalent in regions with different patterns of disease epidemiology vary, and these may determine some of the differences in disease epidemiology in these regions. The epidemiology of viral hepatitis A and E in Mediterranean countries is dynamic and affected by many factors including hygiene, socioeconomic status and vaccination coverage. With its unusual socioeconomic and demographic features, Mediterranean basin is a place with a special interest on the outbreaks of viral hepatitis. Comprehensive epidemiological analysis of Mediterranean cases of HAV and HEV is scarce and few studies have presented in detail the changing epidemiology of HAV and HEV cases. In our review, we evaluate what is known about HAV and HEV outbreaks, using all available publications. Generally, the economic consequences of HEV in the general population are severe, since; testing for HEV infection is not available routinely. With the development of safe and effective hepatitis A vaccines in the early 1990s, understanding hepatitis A epidemiology has taken on new importance, because this information is needed to make well-informed decisions about prevention strategies and appropriate vaccine use. Thereby, both HAV and HEV are a major subject to be taken into account as far as public health is concerned [26].To improve understanding of the epidemiology of HAV and HEV, we conducted a systematic review of outbreaks to describe the distribution and magnitude of all cases in the Mediterranean region occurring during the period 2006- 2017. No prior systematic review was contacted for this time, space and region.

\section{Materials and Methods}

An a priori protocol was performed, based on the PRISMA statement [27], to specify the search strategy, the eligibility criteria, the objectives and the methods of this systematic review. However, this systematic review was not registered with PROSPERO (International prospective register of systematic reviews).

\section{Eligibility criteria}

Not all study design types were included. Single case reports were not included unless the case was reported as the primary infection of the outbreak. This systematic review combined both experimental and non-experimental studies from 2006 onwards. Experts' opinions and qualitative studies were excluded. Also, conference abstracts, editorials and letters to the editor were not included in this systematic review. All HAV and HEV outbreaks dated from 2006 to 2017 were investigated. No publication status restrictions were imposed. All laboratory-confirmed cases or outbreaks, where confirmation was performed by officially indicated methods (molecular, immunoassay, culture microbiological method, etc.), were included. All species of any age were considered. No limits regarding language were applied. All non-English language papers (including French, Spanish, German, Greek and others) were translated by Google translator and were, thus, included in this systematic review. Hand searching authors', experts' or manufacturers' opinions; conference proceedings; editorials and letters to the editors were not included in our study. The selection criteria developed a priori were the following:

- The number of the cases of the outbreak

- $\quad$ Age group of cases

- Symptoms (vomiting, fever, diarrhea, abdominal pain, etc.)

- Country of the epidemic

- Viruses involved in the outbreak (HAV, HEV)

- Place of viral infection

- Method of analysis (molecular, immunoassay, culture microbiological method, etc.)

- Vehicle

- State of the species (fresh/frozen)

- Country of vehicle production

\section{Information sources and search strategy}

Data search was run from November 1, 2016 to December 31, 2017 to scrutinize all the available literature. Peer-reviewed articles concerning HAV and HEV were identified through an assiduous search applied to the electronic databases: Scopus, PubMed, ISI Web of Science Core Correlation and SpringerLink electronic journal. PubMed was selected to be our starting point of research. The search terms used to investigate the reported databases were hepatitis virus", "hepatitis A", "hepatitis E”, “outbreak", "Mediterranean" "outbreaks AND hepatitis," "HAV virus", "HEV virus," "infection AND hepatitis A and E viruses," "hepatitis A and E outbreaks" and "imported cases AND HAV, HEV" published from 2010 to 2017. The search queries were set to include the above terms in (a) article titles, abstract and keywords as for PubMed database, (b) topic 
$(\mathrm{kW})$ and title as for Web of Science Core Correlation and (c) all fields as for Scopus/MEDLINE and SpringerLink. A full electronic search strategy utilizing the Web of Science Core Correlation database is presented in table 1 .

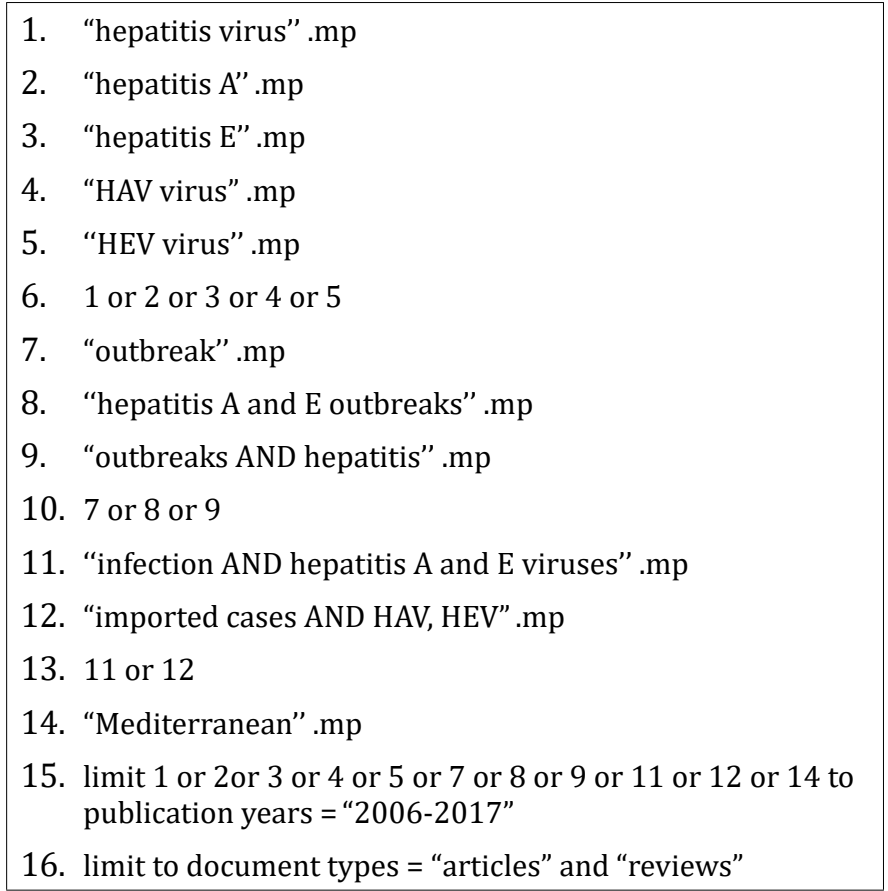

Table 1: Search strategy (Web of Science Core Correlation), conducted in December 2017.

Study selection

An eligibility assessment process was performed, independently, in a standardized manner, mainly by two reviewers (E. Chralambous and I. P. Chatziprodromidou) to evaluate and confirm relevant data to the topic reviewed. A third reviewer also completed the same procedure and agreed with the results (A. Vantarakis). Any disagreements were resolved by a discussion among all authors, leading to a final consensus.

\section{Data collection process and items}

A data extraction sheet was developed, based, mainly, on the rationale proposed by Cochrane Consumers and Communication Review Group 21. Initially, this was pilot-tested in the first 5 articles selected to be included in our study and it was refined according to the needs that emerged. The first author (EC) extracted the data from the studies selected to meet the eligibility criteria set and the second author (IPC) supervised the extracted information. Duplicate publications were identified and considered only once, with the aid of Mendeley, a free reference manager and research paper organizer. No final disagreements between authors ever remained. The information extracted from the articles selected are summarized in table 2 .

\section{Assessment of risk of bias in included studies}

To explore variability in study results (heterogeneity), we specified the following hypotheses before conducting the analysis: the

\begin{tabular}{|c|c|}
\hline Data & Range \\
\hline Year published & $2006-2017$ \\
\hline $\begin{array}{l}\text { Number of samples } \\
\text { taken/susceptible }\end{array}$ & $1-9$ \\
\hline $\begin{array}{l}\text { Number of cases of } \\
\text { HAV,HEV }\end{array}$ & $1-443$ \\
\hline Surveillance program & Yes/no \\
\hline Vaccination program & Yes/no \\
\hline Age of species (years) & $0.3-84$ \\
\hline \multirow[t]{9}{*}{ Epidemiological unit } & Hospitals \\
\hline & School \\
\hline & Cruise Ship \\
\hline & Hotel \\
\hline & Wedding \\
\hline & Nursery \\
\hline & Cafeteria \\
\hline & Institution \\
\hline & Mixed \\
\hline \multirow[t]{6}{*}{ Type of samples } & Pate \\
\hline & Shellfish \\
\hline & Semidried tomatoes \\
\hline & Piglet stuffing \\
\hline & Pomegranate arils \\
\hline & Exotic fruits \\
\hline \multirow[t]{4}{*}{ Symptoms } & Gastrointestinal symptoms \\
\hline & Jaundice \\
\hline & Dead \\
\hline & Not mentioned \\
\hline \multirow[t]{4}{*}{ Method of testing } & Molecular \\
\hline & Immunoassay \\
\hline & Culture \\
\hline & Microbiological method \\
\hline
\end{tabular}

Table 2: Data sheet extracted from articles and records included in this systematic review.

effect size may vary according to the methodological quality of the studies. The quality assessment was done using the QUADAS an evidence based tool [29]. As with all research studies, the whole format of design and conduct of reviews may introduce biases that may affect the systematic review findings. Two reviewers (EC, IPC), independently and in a blind manner, assessed the quality of the included studies. A third reviewer (AV) also evaluated the studies that were selected to be included in this research, and no studies were excluded. The quality assessment of each included study was based on McMaster Critical Review Form for quantitative studies [30] accompanied by a concrete guideline [31]. All included studies were assessed based on three criteria: (a) sample: this was evaluated in order for the selection bias to be reduced (i.e. if the sample size tested was representative of the studied population), the sample size to be sufficient and according to the characteristics of 
the participants, (b) measurement: this was assessed relatively to measurement bias being minimized and (c) analyses: these were evaluated concerning the properness of the analysis followed to answer the research question (i.e. statistical significance) Those three criteria were evaluated and scored as "a": no criteria were met within this component, "b": only some of the criteria met within this component, "c": all criteria were met within this component and "unclear": no data provided.

\section{Results and Discussion}

\section{Study selection - Search results}

Figure 1 describes the procedure of literature selection. We identified 540, 288, 276 and 159 articles from PubMed, Scopus, SpringerLink and Web of Science Core Correlation, respectively, and reviewed articles obtained by screening references. The number of articles was 544 after removing duplicates. Out of the 544 articles, we synthesized 31 original research articles that provide relevant information while excluding the other 513 articles for the following reasons: 1 . Non-Mediterranean populations $(n=503) 2$. Review articles $(n=10)$.

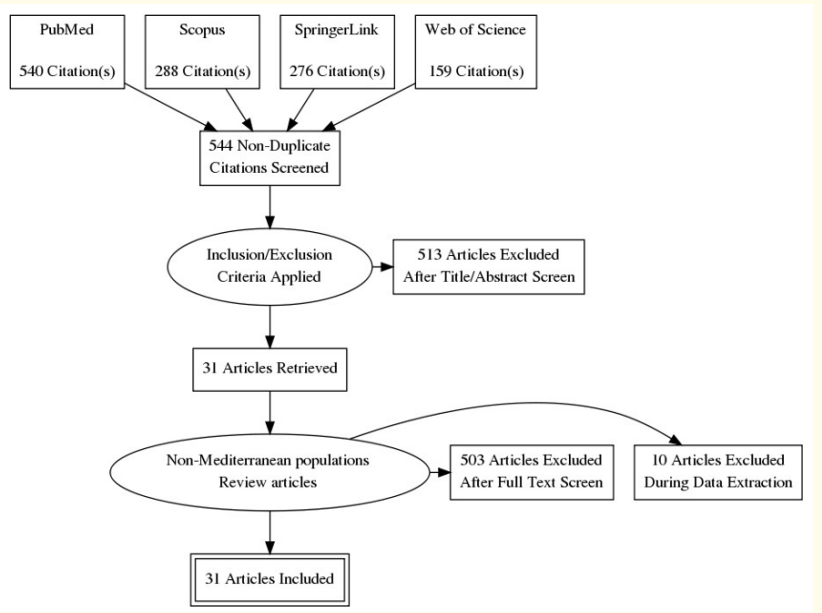

Figure 1: Flow of information through the different phases of this systematic review.

Totally 31 articles met the inclusion criteria, one of which was not in English (Greek). All these studies fulfilled the criteria for case definition of hepatitis A and E virus infection outbreaks. Based on each set of criteria, the number of enrolled individuals was recorded (if this information was provided in the article). Characteristics of included studies.

The greatest number of studies $(5,5.2 \%)$ occurred in years 2013, 2008 and 2006 while 4 (4.2\%) occurred in 2016 and 2010, 3 (3.1\%) in 2007 and 1 (1.0\%) in 2017, 2014, 2012, 2011 and 2009, respectively (Figure 2). Regarding the "country of epidemics", we estimated that the largest number of outbreaks ( 9 outbreaks, 9.4\%) occurred in France, while 5 outbreaks (5.2\%), occurred in
Spain, 4 outbreaks (4.2\%) occurred in Egypt and Italy, 3 outbreaks (3.1\%) occurred in Israel, 2 outbreaks (2.1\%) occurred in Greece and 1 outbreak (1.0\%) occurred in Turkey, Algeria, Morocco and Tunisia respectively. Among all outbreaks (31) included in this systematic review, 10 (32.3\%) occurred in hospitals, 8 did not mention the place that outbreak occur, 5 outbreaks (16.1\%) occurred in mixed locations, 2 outbreaks (6.5\%) in schools and 1 outbreak (3.2\%) in cruise ship, hotels, wedding locations, nursery school's cafeteria and institution (Figure 3).

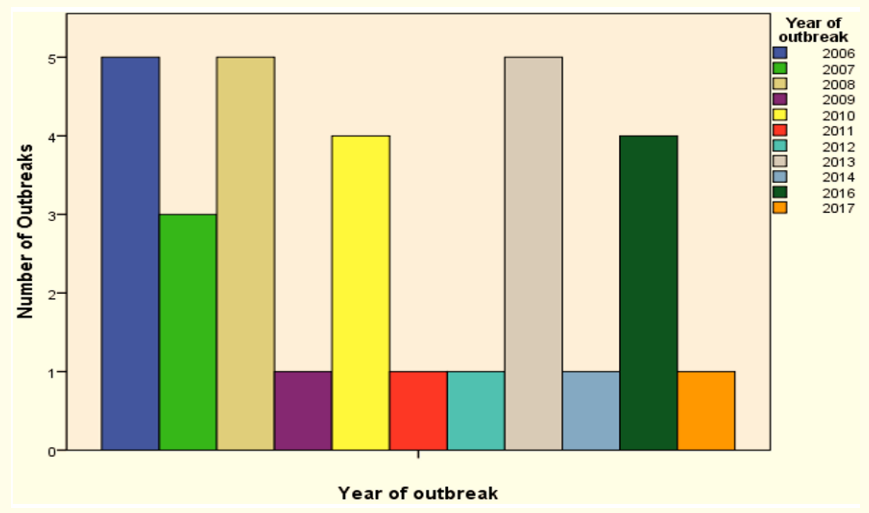

Figure 2: Number of viral hepatitis (HAV, HEV) outbreaks upon year (2006-2017).

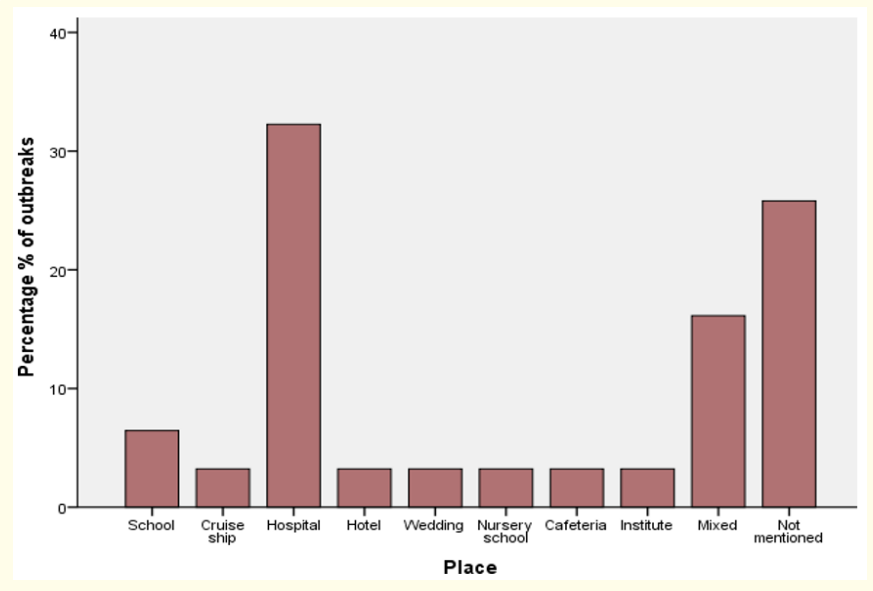

Figure 3: Viral hepatitis (HAV, HEV) outbreaks upon places.

The age group of those who were sick was between 0.3 and 84 years old.

Thirty outbreaks (96.8\%) recorded general gastrointestinal symptoms (vomiting, nausea, anorexia and abdominal pain), typical of HAV and HEV, while only 1 outbreak (3.2\%) did not mention any symptomatology. In 6 outbreaks (19.4\%) jaundice was outlined as major symptom, while 25 outbreaks (80.6\%) mentioned no jaundice. Twelve outbreaks (38.7\%, 1.111 cases) mentioned hospitalization while the other 19 outbreaks (61.3\%, 1.987 cases) 
did not mention anything. Only 1 outbreak (3.2\%, 443 cases) had mentioned deaths while 11 (38.7\%,467 cases) mentioned that no deaths had occurred and 19 (61.3\%, 2.190 cases) failed to mention anything about deaths (Figure 4). The sample type tested was missing in all the outbreaks.

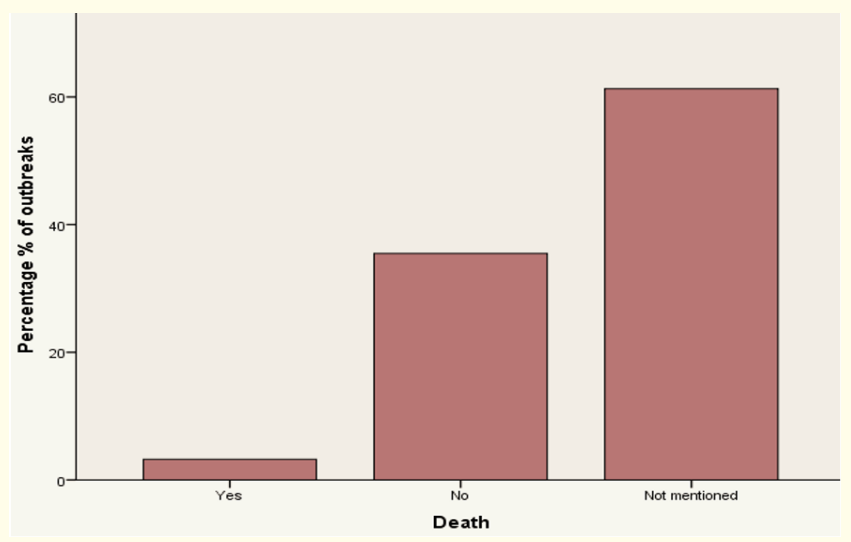

Figure 4: Outbreaks of viral hepatitis (HAV, HEV) were death occurred

Twenty (64.5\%) of the diagnosis were performed both epidemiologically and, in the laboratory, while 8 (25.8\%) were done only epidemiologically and the other $5(9.7 \%)$ outbreaks did not refer to the way of diagnosis (Figure 5).

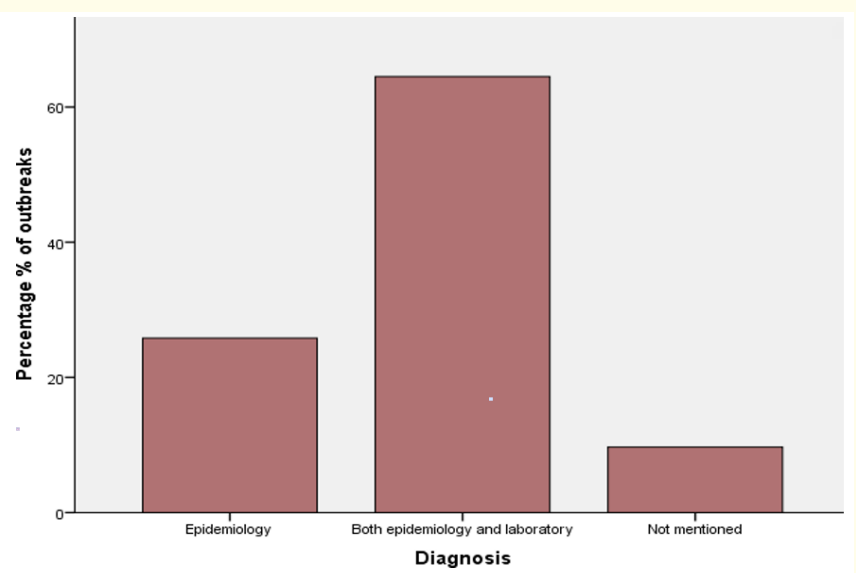

Figure 5: Viral hepatitis (HAV, HEV) distribution upon diagnosis.

Information regarding "species of fresh produce" in relation to the outbreak area was missing in 20 outbreaks (64.5\%). Also, 21 outbreaks (67.7\%) failed to mention the state of the species (frozen or fresh). Only $6(19.4 \%)$ mentioned that the species were fresh and the other $4(12.9 \%)$ mentioned that the species were frozen (Figure 6). However, the most frequent species of fresh produce were the berries in $6(12.9 \%)$ of the cases the other 1 (3.2\%) were pate, shellfish, semidried tomatoes, piglet stuffing, pomegranate arils and exotic fruits (such as mangos and strawberries) respectively. The country of produce which had the most outbreaks was France with 7 (25.9\%) then Spain and Italy with 4 (14.8\%), then Egypt and Israel with $3(11.1 \%)$, then Greece with 2 (7.4\%) and finally Turkey, Algeria, Morocco and Tunisia with 1 (3.7\%) respectively (Figure 7).

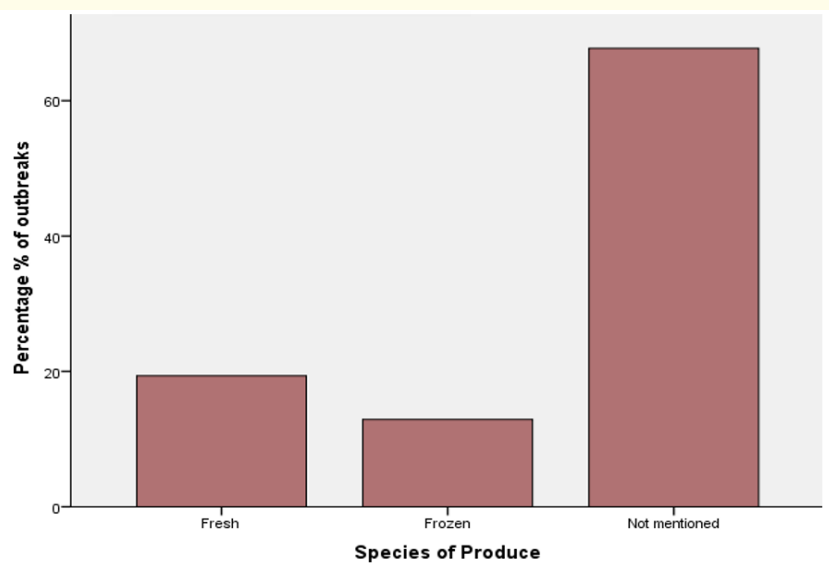

Figure 6: Viral hepatitis (HAV, HEV) outbreaks upon species of produce.

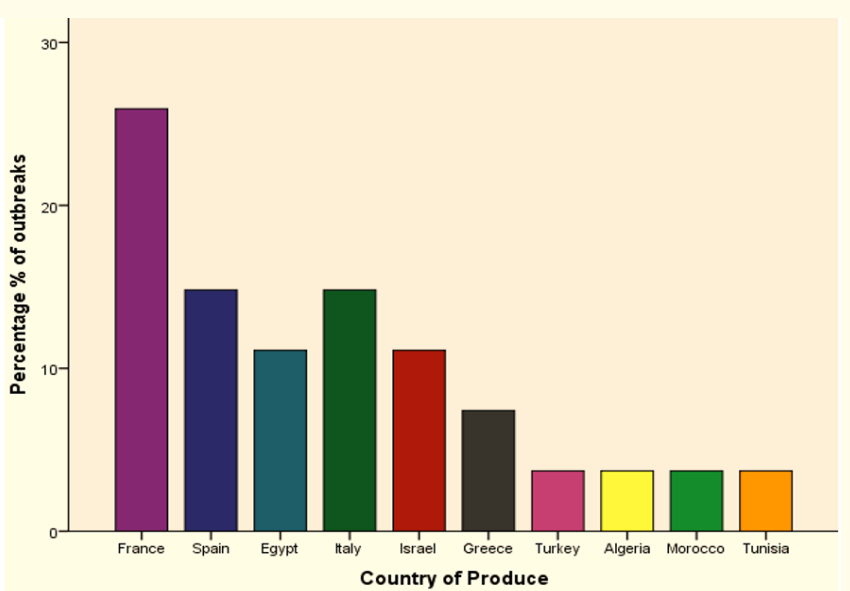

Figure 7: Distribution of viral hepatitis (HAV, HEV) outbreaks in countries of produce.

\section{Discussion}

In our systematic review, a Mediterranean dataset spanning almost 12 years was systematically collated to search and investigate the epidemiological profile of HAV and HEV. Our findings suggest that both viruses still exist and expand, and this could be related to global spread of the virus via population immigration. In the last decade, outbreaks of hepatitis A and E have been reported from several areas with warfare and conflict, causing human displacement. Several large HAV and HEV outbreaks, involving hundreds to thousands cases, were reported from refugee camps [32-35]. Other major outbreaks occurred in more-restricted (closed) set- 
tings, such as military units [36,37], colleges, prisons, and factories $[37,38]$.

Regarding the geographical extent of outbreaks, in the Mediterranean basin, France holds the lead [39]. South Mediterranean countries have reported some but not of great importance outbreaks during 2006-2017.

Our findings agree with those of Shaw and Erdogan., et al. which conclude that the incidence of the virus varies with age, race, ethnicity and geographic region $[40,41]$. HEV is a major cause of epidemic waterborne hepatitis in tropical and subtropical countries, particularly in areas with poor sanitary conditions. The infection is endemic to the south- east and central Asia, the Middle East, and northern and western Africa $[42,43]$. In endemic countries, HEV is a major cause of acute sporadic hepatitis as well as epidemic outbreaks of hepatitis related to faecal contamination of drinking water during heavy rainfall or floods [42]. In no endemic regions such as North America, Western Europe, and developed countries of the Asia-Pacific, HEV is an infrequent cause of acute hepatitis and is usually thought to be acquired during travelling to diseaseendemic areas, although autochthonous (locally acquired) HEV infection is now increasingly recognised [44].

The seroprevalence of anti-HEV in Egypt is among the highest reported throughout the world, exceeded the $60 \%$ in the first decade of life, peaked at $76 \%$ in the second decade and remained above $60 \%$ until the eighth decade. In the Nile Delta, the seroprevalence of anti-HEV was $17.2 \%$ and increased by age [45]. Çetinkaya., et al. reported anti-HEV seropositivity as $7.6 \%$ in blood donors in Turkey [46]. It is sporadic (2.1\%) in Europe. In a survey covering 5 regions of Turkey the prevalence rate of anti-HEV in the general population was found to be $5.9 \%$. The prevalence rates reported from other countries were $1.1 \%$ in Netherlands [47], 1.7\% in Italy [48], 7.3\% in Germany [24].

Immunoprophylaxis for HEV is not available and proper sanitation is the most salient way of prevention. According to another systematic review, where the global burden of hepatitis E outbreaks was analysed, most of the studies reported faecal contamination of drinking water as the major route of transmission during HEV outbreak [37]. The most commonly reported underlying cause of this contamination was leakage of water pipeline distribution system, either due to damaged or poor construction. As the water pipelines located close to drain or sewerage system, the damaged facilitate mixing of sewage materials and drinking water supplied to the household, causing water-borne outbreaks such as HEV, HAV, shigellosis and cholera [37]. We agree with their suggestion that, this kind of outbreaks can be prevented by proper construction of water pipelines, keeping them away from the drain system, and by monitoring of pipelines for damage. It is clear that public awareness through reports [49], aggressive investigation and case finding by authorities [50] can substantially affect both the number of outbreaks identified and the volume of illness reported [51]. For example, investigations of specific disease out- breaks linked to foodstuff consumption have been reported in the scientific literature in many countries; however, few countries systematically collate and report such data through a disease surveillance system. In the UK, for instance, collection of epidemiological data for England and Wales is performed by the Public Health Laboratory Service Communicable Disease Surveillance Centre and published periodically [52], [53]. Reported incidents, therefore, relate principally to large functions where multiple illnesses are more apparent and where classical statistical correlations between illness and a food vehicle can be made. Individual cases are rarely if ever investigated and do not feature in the disease statistics in any country [51]. Consequently, the actual number of HAV and HEV outbreaks should be much higher than what it is presented in this study. Therefore, the problem of HAV and HEV infections should not be underestimated by national and international health agencies. HAV and HEV represent a significant health problem, especially in the developing countries. Acute sporadic form of HAV and HEV diseases are the most frequent cause of acute viral hepatitis globally [50]. Epidemics of HAV and HEV, either in a small or large scale, occur periodically up to this moment, as reported from India [50]. Our systematic review has several limitations that could be divided into two separate categories: firstly, there are limitations of the methodology of the review and secondly limitations related to the variable quality of the source-articles. The identification of numerous references obtained through the studies' lists distinguished by search engines, i.e. $10 \%$ of all citations included, indicates that search strategy and eligibility criteria set may have been very specific and restrictive. Concerning the source limitations, the surveillance and diagnostic procedures, the recording system applied in each country as well as the state of a country are not the same among the studies evaluated. The quality of each study varies and may lead to inaccurate conclusions. The keywords set in our search strategy were specific and may eliminate the number of articles included. Those keywords concerned article title, abstract or keywords and thus papers missing to mention the above key terms in their titles, abstracts or keywords, albeit reporting them in their full text, may have also been missed. The cases identified in this systematic review are likely to be underestimates of the real incidence of HAV and HEV, due to the limiting timeline of 2006-2017 set by us and difficulties of precisely scrutinize and include data referring to years 2006-2017. There were outbreaks reporting massive numbers of cases for 2001-2005, which didn't separated cases per year and were rejected, because they didn't allow us to "isolate" the number of cases concerning to the timeline set by us (2006-2017). Another main limitation of this systematic review is that there is no surveillance for neither of the diseases (hepatitis A, hepatitis E), while the patient population, the antibiotic regimen and the outcome definitions are not the same across studies. Globalization has made the propagation of diseases like viral hepatitis relatively easy throughout the world. Europe is a lively continent where thousands of people move to seek a job, to find new opportunities or simply for travelling purposes. Moreover wars, natural disasters or political instabilities make the trend for immigration even more justifiable. These events facilitate the spread of hepatitis 
viruses throughout the Mediterranean basin [19,54]. At the other end of the spectrum morbidity and mortality rates related to viral hepatitis are progressively decreasing in this region of Europe. Better hygienic measures, widespread use of disposable syringes, definition of high risk groups, universal vaccination for HAV infection, demographic and behavioural factors may play a role in the reduced prevalence of viral hepatitis [55]. Hepatitis $\mathrm{E}$ is a major cause of viral hepatitis in the Mediterranean. Clinical importance of the other viruses remains to be determined by further studies. The challenges to be faced in the next millennium involve the introduction of new and more antigenic vaccines for hepatitis. Therefore, the long-standing assault of viral hepatitis should eradicate in the region, in order to regain its reputation as a healthy region, seat for eternal peace and felicity. Deficiencies emerging from our review draw attention to key areas that future outbreak reports should try to address. Future studies in this area should aim to record the presence of species that are thought to be involved, while effort should focus on possible routes of transmission. This could allow a greater number of studies to be assessed and included in reviews like this one. Generally, anything that is implicated (weather conditions, water collections etc.) should be clearly stated. The case definition should be clearly stated. It is of great importance to highlight the contingent role of such factors and to encourage researchers to explicitly investigate whether such factors occurred prior to the outbreak or their role after the outbreak.

\section{Conclusion}

This review studied the prevalence of HAV and HEV outbreaks in Mediterranean countries. It also suggests that HAV and HEV outbreaks do occur in both developed and developing countries and this constitutes as an important liability on public health. The outbreaks, the frequency and the patterns of hepatitis viruses depict variations across the Mediterranean region depending on several demographic and socioeconomic factors.

The prevalence of viral hepatitis infection is still high and consists a serious public health challenge. Hepatitis A and E are endemic in the region and despite the subclinical features of the infections among residents, $\mathrm{HAV}$ and HEV conceal a danger to tourists and expatriates visiting the Mediterranean countries. Where an outbreak is reported, some effort should be made to classify the probable route (or routes) of transmission. This would allow a greater proportion of outbreaks to be identified and included in systematic analyses. Where the number of cases involved in an outbreak was reported, the case definition used should be clearly stated. If a climatic event is thought to be implicated in an outbreak, details of how it may have led to contamination of the vehicle should be reported. It is also important to raise awareness of the potential role of such events in viral hepatitis outbreaks, to encourage authors to question explicitly whether such an event occurred prior to the outbreak and if so, to detail it in a structured way. Systematic surveillance and prevention programs should be developed and be enhanced in all countries. Further epidemiological studies are needed to understand the need of mandatory surveillance of both HAV and HEV.

\section{Acknowledgements}

A short acknowledgement section can be written acknowledging the sources regarding sponsorship and financial support. Acknowledging the contributions of other colleagues who are not included in the authorship of this paper should also be added in this section. If there are no acknowledgements, then this section need not be mentioned in the paper.

\section{Conflict of Interest}

\section{Declarations}

Ethics approval and Consent to Participate

Not applicable.

\section{Consent for Publication}

Not applicable.

\section{Availability of Data and Material}

The datasets used and/or analysed during the current study are available from the corresponding author on reasonable request.

\section{Competing Interests}

The authors declare that they have no competing interests.

\section{Funding}

Not applicable.

\section{Bibliography}

1. Regev A and Schiff ER. "Viral hepatitis A, B, and C". Clinical Liver Disease 4 (2000): 47-71.

2. Franco E., et al. "The evolution of the burden of viral hepatitis from 1990 to 2013: still an open challenge to global public health policy". Hepatobiliary Surgery and Nutrition 6 (2017): 277-279.

3. Bellou M., et al. "Shellfish-Borne Viral Outbreaks: A Systematic Review". Food and Environmental Virology 5 (2013): 13-23.

4. Chatziprodromidou IP., et al. "Global avian influenza outbreaks 2010-2016: a systematic review of their distribution, avian species and virus subtype". Systematic Reviews 7 (2018): 17.

5. Brachman PS. "Control of Communicable Diseases Manual, 17th Edition". American Journal of Epidemiology 154 (2001):783-a-784.

6. Bosch A Albert BN. "Human viruses in water". Elsevier (2007).

7. Elamri DE., et al. "Detection of human enteric virusesin shellfish collected in Tunisia". Letters in Applied Microbiology 426.43 (2006): 399-404. 
8. Cheng MS and Toh C-S. "Novel biosensing methodologies for ultrasensitive detection of viruses". Analyst 138 (2013): 6219.

9. Ueki Y., et al. "Norovirus pathway in water environment estimated by genetic analysis of strains from patients of gastroenteritis, sewage, treated wastewater, river water and oysters". Water Research 39 (2005): 4271-4280.

10. Boxman ILA. "Human Enteric Viruses Occurrence in Shellfish from European Markets". Food and Environmental Virology 2 (2010): 156-166.

11. Rodríguez-Lázaro D., et al. "Virus hazards from food, water and other contaminated environments". FEMS Microbiology Reviews 36 (2012): 786-814.

12. Goyal SM and Cannon JL. Viruses in foods (2017).

13. Miura T., et al. "Detection of Hepatitis E Virus in Sewage After an Outbreak on a French Island". Food and Environmental Virology 8 (2016): 194-199.

14. Poovorawan Y., et al. "Epidemiology and prophylaxis of viral hepatitis: A global perspective". Journal of Gastroenterology and Hepatology (2002): 17.

15. Consortium TH., et al. "Hepatitis A virus in urban sewage from two Mediterranean countries". Epidemiology and Infection (2007): 270-273.

16. Jeong SH and Lee HS. "Hepatitis A: clinical manifestations and management”. Intervirolog 53.1 (2010): 15-19.

17. Couturier E., et al. "Rapid communications Cluster of cases of hepatitis A with a travel to Egypt". France History (2008): 46-48.

18. Bernard H and Frank C. "Rapid communications Cluster of cases of hepatitis A among travellers returning from Egypt". Germany (2008): 2-3.

19. Tahan V., et al. "Epidemiology of viral hepatitis in the Mediterranean basin". Roczniki Akademii Medycznej w Bialymstoku 48 (2003): 11-17.

20. Kurugol Z., et al. "Changing epidemiology of hepatitis A infection in Izmir". Turkey. Vaccine. Elsevier Ltd 29 (2011): 62596261.

21. Bader TF. "Hepatitis A vaccine". The American Journal of Gastroenterology 91 (1996): 217-222.

22. Lopalco Pier Lssbcgmbmbád. "Hepatitis A and B in children and adolescents - what can we learn from Puglia (Italy) and Catalonia (Spain)? Vaccine". Elsevier 19 (2000): 470-474.

23. Report WHO. "Hepatitis E vaccine: WHO position paper, May 2015 - Recommendations". Vaccine. Elsevier Ltd 34 (2016): 304-305.

24. Wang Y. Hepatitis E virus (2018).
25. Mirazo S., et al. "Transmission, diagnosis, and management of hepatitis E: an update". Hepatic Medicine: Evidence and Research 6 (2014): 45-59.

26. Kim S-E., et al. "Determination of fecal shedding rates and genotypes of swine hepatitis E virus (HEV) in Korea". Journal of Veterinary Medical Science 70 (2008): 1367-1371.

27. Liberati A., et al. Guidelines and Guidance The PRISMA Statement for Reporting Systematic Reviews and Meta-Analyses of Studies That Evaluate Health Care Interventions: Explanation and Elaboration (2008).

28. Whiting PF., et al. "QUADAS-2: A Revised Tool for the Quality Assessment of Diagnostic Accuracy Studies". Annals of Internal Medicine 155.8 (2011): 529-536.

29. Letts L., et al. "Guidelines for critical review form - Qualitative studies (version 2.0)". McMaster University Occupational Therapy Evidence-Based Practice Research Group (2007).

30. Law M., et al. Guidelines for Critical Review of Qualitative Studies Based on Guidelines for Critical Review Form-Qualitative Studies (2018).

31. Ahmed JA., et al. "Hepatitis E outbreak, Dadaab refugee camp, Kenya, 2012”. Emerging Infectious Diseases 19.6 (2013):10101012.

32. Boccia D., et al. "High mortality associated with an outbreak of hepatitis E among displaced persons in Darfur, Sudan". Clinical Infectious Diseases 42 (2006): 1679-1684.

33. Thomson H., et al. "Housing improvements for health and associated socio-economic outcomes". The Cochrane Database of Systematic Reviews 28 (2013): CD008657.

34. Reverbel T. "Epidemiology of Hepatitis A and Hepatitis E in Brazil". (2014): 19-21.

35. Kumar N., et al. "Emerging biosensor platforms for the assessment of water-borne pathogens". Analyst (2018).

36. Hakim MS., et al. "The global burden of hepatitis E outbreaks: A systematic review". Liver International (2016): 1-13.

37. Pérez-Gracia MT., et al. "Hepatitis E: Current status". Reviews in Medical Virology (2013): 384-398.

38. Herriman R. France reports nearly 800 hepatitis A cases in first five months of 2017 -Outbreak News Today (2017).

39. Shaw F. "Epidemiology of Hepatitis A in the United States". Viral Hepatitis 6 (1997):14-15.

40. Erdoğan MS., et al. "The epidemiology of hepatitis a virus infection in children, in Edirne, Turkey". European Journal of Epidemiology 19 (2004): 267-273.

41. Suay B., et al. Hepatitis E: Latest developments in knowledge Hepatitis E: latest developments in knowledge (2016). 
42. Manka P., et al. "Hepatitis E Virus Infection as a Possible Cause of Acute Liver Failure in Europe". Clinical Gastroenterology and Hepatology 13 (2015): 1836-1842.

43. Report WHO. "Hepatitis E vaccine: WHO position paper, May 2015 - Recommendations". Vaccine Elsevier Ltd 34 (2016): 304-305.

44. Corbellini LG., et al. "Effect of slaughterhouse and day of sample on the probability of a pig carcass being Salmonella-positive according to the Enterobacteriaceae count in the largest Brazilian pork production region". International Journal of Food Microbiology 228 (2016): 58-66.

45. Çetinkaya B., et al. "Evaluation of pediatric patients with hepatitis A". Journal of Infection in Developing Countries 8 (2014): 326-330.

46. Hogema BM., et al. "Incidence and duration of hepatitis e virus infection in Dutch blood donors". Transfusion 56 (2016): 722-728

47. Henrique Paiva H., et al. "Molecular Characterization of Swine Hepatitis E Virus from Southeastern Brazil". Brazilian Journal of Microbiology 38 (2007): 693-698.

48. Chandnani M., et al. "A case report about the most common yet most forgotten hepatitis E". American Journal of Case Reports 17 (2016): 584-586.

49. Hakim MS., et al. "The global burden of hepatitis E outbreaks: A systematic review". Liver International (2016).

50. Bellou M., et al. "Shellfish-Borne Viral Outbreaks: A Systematic Review". Food and Environmental Virology 5 (2013): 13-23.

51. Sockett PN., et al. "Foodborne disease surveillance in England and Wales: 1989-1991". Communicable Disease Report CDR Review 3 (1993): R159-R173.

52. Patterson' T., et al. "Outbreak of SRSV gastroenteritis at an international conference traced to food handled by a post-symptomatic caterer". Epidemiology and Infection 111 (1993): 157-162.

53. NVN Naoumov N V. "Hepatitis A and E. Medicine (Baltimore)". (2007): 35-38.

54. El Sayed Zaki M., et al. "Clinicolaboratory study of mother-toneonate transmission of hepatitis E virus in Egypt". American Journal of Clinical Pathology 140 (2013): 721-726.

Volume 2 Issue 11 November 2019

(C) All rights are reserved by Elena Charalambous., et al. 